\title{
Ultrasound-guided percutaneous injection of methylene blue to identify nerve pathology and guide surgery
}

\author{
Joseph A. Osorio, MD, PhD, Jonathan D. Breshears, MD, ${ }^{1}$ Omar Arnaout, MD, ${ }^{2}$ \\ Neil G. Simon, MBBS, FRACP, ${ }^{3}$ Ashley M. Hastings-Robinson, BA, ${ }^{2}$ Pedram Aleshi, MD, ${ }^{4}$ and \\ Michel Kliot, MD²
}

\begin{abstract}
1Department of Neurological Surgery, University of California, San Francisco, California; 2Department of Neurological Surgery, Northwestern University Feinberg School of Medicine, Chicago, Illinois; ${ }^{3}$ Prince of Wales Clinical School, University of New South Wales, Randwick, and Department of Neurology, St. Vincent's Hospital, Darlinghurst, New South Wales, Australia; and ${ }^{4}$ Department of Anesthesia \& Perioperative Care, University of California, San Francisco, California
\end{abstract}

\begin{abstract}
OBJECT The objective of this study was to provide a technique that could be used in the preoperative period to facilitate the surgical exploration of peripheral nerve pathology.

METHODS The authors describe a technique in which 1) ultrasonography is used in the immediate preoperative period to identify target peripheral nerves, 2) an ultrasound-guided needle electrode is used to stimulate peripheral nerves to confirm their position, and then 3) a methylene blue (MB) injection is performed to mark the peripheral nerve pathology to facilitate surgical exploration.
\end{abstract}

RESULTS A cohort of 13 patients with varying indications for peripheral nerve surgery is presented in which ultrasound guidance, stimulation, and MB were used to localize and create a road map for surgeries.

CONCLUSIONS Preoperative ultrasound-guided MB administration is a promising technique that peripheral nerve surgeons could use to plan and execute surgery.

http://thejns.org/doi/abs/10.3171/2015.6.FOCUS15220

KEY WORDS ultrasonography; peripheral nerve; methylene blue

$\mathrm{P}$ ERIPHERAL nerve surgical targets are identified using a combination of preoperative imaging and intraoperative anatomical landmarks. Although this allows for successful localization of peripheral nerve lesions, it often involves extended surgical exposures with longer operative times and a related increase in morbidity. Surgeries should aim to optimize efficiency, accuracy, and safety to improve outcomes and minimize harm to the patient. In this study, we describe a technique in which 1) ultrasonography is used in the immediate preoperative period to identify target peripheral nerves, 2) an ultrasound-guided needle electrode is used to stimulate peripheral nerves to confirm their position, and then 3) a methylene blue (MB) injection is performed to mark the peripheral nerve pathology to facilitate surgical exploration.

Ultrasonography is a powerful tool that can provide the surgeon with real-time dynamic visualization of periph- eral nerves and critical surrounding structures, and potentially provide insight on the underlying pathology. ${ }^{11,14,20}$ Compared with other navigation technology such as intraoperative MRI and CT, ultrasound has the advantage of being portable, cost-effective, and radiation-free (compared with CT). Ultrasound guidance is an inexpensive and widely available technology that provides dynamic imaging that can be easily incorporated into the operating room. ${ }^{1,4,7,11,20}$ Furthermore, several studies have confirmed the accuracy of ultrasonography in identifying peripheral nerves and its usefulness for guidance for the peripheral nerve surgeon. $5,10,12,13,16$

The use of MB, also referred to as supravital staining in the literature, is a well-known technique in the field of anatomical study. ${ }^{9}$ On the surgical frontier, MB has been incorporated into the operating room safely by surgeons of various specialties to improve identification of vital 
anatomical structures. ${ }^{6,9,19}$ For instance, the use of MB has been reported during parotid gland surgery, where it helps guide tumor resection as an indirect identifier of the facial nerve. ${ }^{17}$ In addition, MB has been used to directly stain critical nerves to avoid injury during surgical procedures. ${ }^{18}$ Finally, anesthesiologists routinely use cannulated needles for delivery of nerve blocking agents, and often do so with ultrasound guidance, electrical nerve stimulation guidance, or both..$^{15}$ However, the combined use of these techniques has not been previously reported, namely the percutaneous placement of a cannulated needle filled with MB under ultrasound and electrical nerve stimulation guidance, to deliver a marker to create a colorized surgical corridor from the skin surface to the deeper lying target structure. ${ }^{6}$ The technique presented provides a novel approach for the peripheral nerve surgeon to plan and execute surgery expeditiously and efficiently through an incision that is optimally placed and sized so as to provide direct and adequate exposure while minimizing collateral damage to surrounding tissues and structures.

\section{Methods}

A cohort of 13 patients with varying indications for peripheral nerve surgery was included in this study (Table 1). A neurophysiologist was involved in all cases for monitoring of both indirect and direct nerve stimulation.

The patients were brought to the operating room and placed under general anesthesia. Prior to skin preparation, an anesthesiologist (P.A.) who was experienced in ultrasound neurography performed the sonographic nerve mapping. A Mindray M7 portable ultrasonic imaging system was used with a linear array transducer (L14-6s, nominal frequency range 6-14 MHz; or 7L4s, nominal frequency range 5-10 MHz). Once the target nerve was identified, a 22-gauge, $50-\mathrm{mm}$ insulated needle or a 21-gauge, $10-\mathrm{mm}$ insulated needle (SonoPlex Stim cannula, Pajunk) was inserted in plane with the transducer while the target nerve was kept in the field of view (Fig. 1A). The needle was inserted with the nerve stimulator set at $1.0 \mathrm{~mA}$ at $2 \mathrm{~Hz}$ (Fig. 1B). Accurate placement of the needle tip relies on ultrasound guidance relative to the nerve, as well as direct stimulation of the nerve and recording electromyography responses from electrodes placed in target muscles (Fig. 1C), which was captured and recorded by the neurophysiologist on a monitor (Fig. 1D and E). A plantar flexion foot motor response was also elicited and could be seen and palpated, providing additional confirmation of accurate placement of the needle tip (Fig. 1C).

Once the 22-gauge hypodermic needle was well positioned, $0.5 \mathrm{ml}$ of a dilute solution of MB was subsequently injected to demarcate the distal neural structures (Fig. 2D). An additional 3-4 $\mathrm{ml}$ of MB was subsequently injected as the needle was slowly withdrawn to provide a colorized operative corridor to the target nerve (Fig. 2C). A dilute and safe concentration of $\mathrm{MB}$ was used at a low volume that required no consent. The needle was then removed and the field prepared and draped in the usual sterile fashion. A skin incision was made over the needle puncture site, and the tract highlighted by the MB dye guided the operative corridor to the nerve of interest and surgical tar-
TABLE 1. Patients who underwent intraoperative ultrasound-guided needle injections with MB

\begin{tabular}{ccll}
\hline $\begin{array}{c}\text { Case } \\
\text { No. }\end{array}$ & Revision & \multicolumn{1}{c}{ Pathology } & \multicolumn{1}{c}{ Nerve } \\
\hline 1 & No & Piriformis syndrome & Sciatic \\
\hline 2 & No & Nerve tumor & Sciatic \\
\hline 3 & No & Nerve tumor & Infrapatellar \\
\hline 4 & Yes & Transposed nerve re-explora- & Ulnar \\
& & tion & \\
\hline 5 & Yes & Nerve laceration & Ulnar \\
\hline 6 & No & Extraneural ganglion cyst & Radial/ulnar/median \\
\hline 7 & No & Myxoma & Sciatic \\
\hline 8 & No & Lipoma & Radial/ulnar/median \\
\hline 9 & Yes & Nerve graft exploration & Ulnar \\
\hline 10 & No & Nerve tumor & Sciatic \\
\hline 11 & Yes & Nerve graft exploration & Median \\
\hline 12 & Yes & Nerve laceration & Median \\
\hline 13 & No & Exploration & Lateral femoral cut \\
\hline
\end{tabular}

get. The operation proceeded in the usual fashion at this point.

\section{Results}

In all cases, the preoperative ultrasonography easily identified the nerve (Fig. 2B) and region of interest (Fig. $2 \mathrm{~A})$. Indirect stimulation of the target nerve, while maintaining direct ultrasound visualization, was confirmed prior to the injection of MB in all cases. An MB-colored surgical corridor was created in all cases, which allowed the surgeon to dissect with confidence toward the target structures quickly and efficiently while minimizing collateral trauma to surrounding tissues. Examples of operative dissections with $\mathrm{MB}$ staining are shown in Fig. 2C and Fig. 3C and described below. Methylene blue dye injections were always extraneural and typically stained the epineurium.

There were no cases in which patients experienced an allergic reaction to the $\mathrm{MB}$ injection. The needle used for nerve stimulation and for administration of the MB did not cause any complications, such as nerve injury or hematoma. In every surgery, the MB was present throughout the dissection, and it was useful for operative exposure. There were no patients that experienced permanent skin discoloration.

\section{Illustrative Cases Case 1}

A 42-year-old man presented after having 7 years of left buttock pain, which was exacerbated with pressure (primarily sitting) and relieved by standing. The pain was unresponsive to physical therapy. His workup included an electromyogram that showed chronic reinnervation changes that were consistent with left sciatic neuropathy. He also received an MR neurogram, which was unrevealing (Fig. 2A). He did receive a treatment of botulinum toxin, which served as supporting evidence for left piri- 


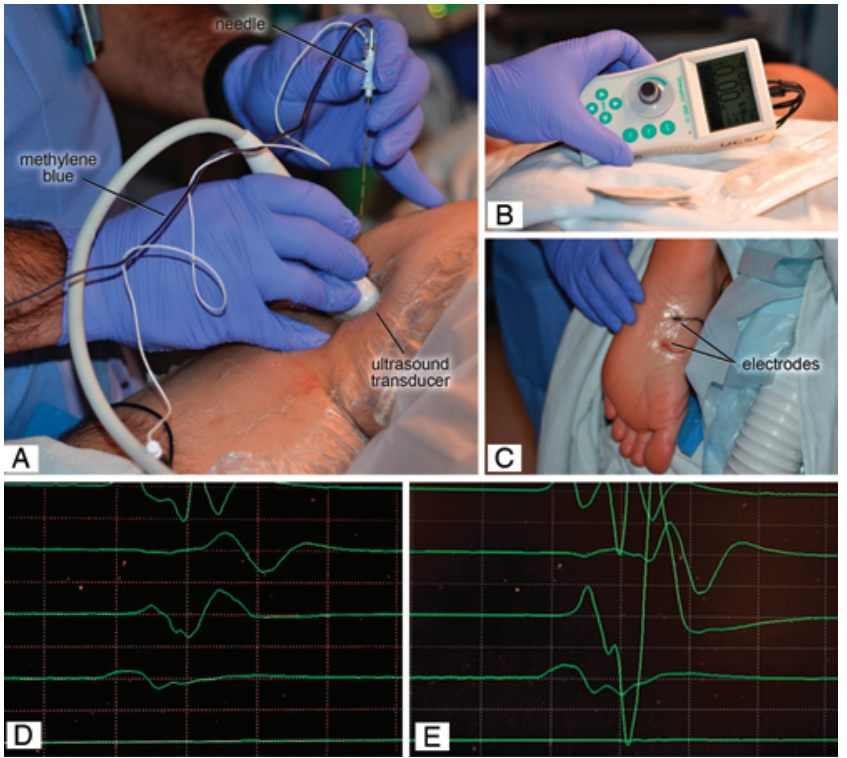

FIG. 1. Images demonstrating the intraoperative administration of MB delivery using ultrasound image guidance along with nerve stimulation. A: The ultrasound transducer is over the operative region of interest, and the cannulated stimulation needle is shown just prior to advancement into the skin. B: The stimulation needle has 2 wires, one that is connected to the transducer $(B)$, and the other that is labeled showing the MB $(A)$. C: Electrodes are placed on the target muscle group that is controlled by the selected nerve; the anode and cathode are shown. Also shown is the assistant both looking and palpating for movement in the plantar flexor foot muscles as the stimulation is increased. D and E: Shown are 2 screen shots of the muscle groups monitored by the neurophysiologist, demonstrating nerve stimulation. As the needle is advanced closer to the nerve of interest, the amplitude of the signal becomes detectable (D), and the closer the stimulation needle comes in proximity to the nerve, the larger the amplitude is recorded $(E)$.

formis syndrome. On physical examination, he had full motor strength, normal sensation, normal reflexes, and a negative straight leg raise test. He had focal tenderness to deep palpation over his left sciatic notch and maneuvers causing stretching of the piriformis muscle elicited deep left buttock pain.

Preoperatively, ultrasonography was used to identify the sciatic nerve, and MB was administered as described above (Fig. 2B). On surgical exploration, a vertical incision measuring $8 \mathrm{~cm}$ in length was centered about the needle site (Fig. 2C). The trail of MB was followed all the way to the piriformis muscle and the underlying sciatic nerve, which was identified using direct electrical stimulation to both its medial tibial and lateral peroneal components (Fig. 2D). It was noted during the operation that a fibrotic portion of the piriformis muscle was compressing the underlying proximal left sciatic nerve. Subsequently, the left sciatic nerve was fully decompressed both proximally and distally. Postoperatively the patient experienced a dramatic reduction in his left buttock pain.

\section{Case 7}

A 52-year-old woman presented with a 3-year history of progressively worsening sciatica. She had also noted that her right buttock was tender to pressure. She underwent
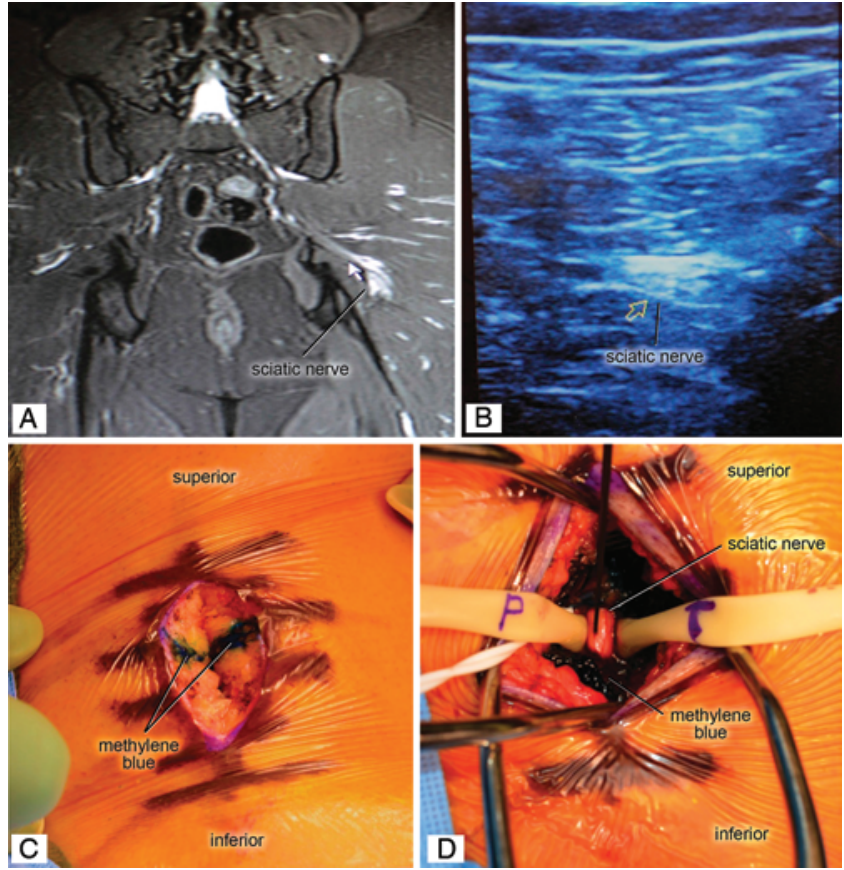

FIG. 2. Case 1. A case example is shown of a patient who presented with left buttock pain that was exacerbated by direct pressure and was diagnosed with left piriformis syndrome. A: The MR neurogram is shown, and labeled is the region of interest showing the sciatic nerve (arrow). B: An intraoperative ultrasound image is shown that was acquired at the time of administration of MB with the sciatic nerve (arrow) labeled as the target of interest. C and D: Operative photographs from the decompression performed on the patient with piriformis syndrome. Shown is the initial subcutaneous dissection demonstrating the MB tract that is followed through a small operative window to locate the sciatic nerve $(\mathrm{C})$. Methylene blue is shown surrounding the sciatic nerve, whose identity was also confirmed using direct electrical stimulation of the nerve while recording electromyography responses from appropriate muscles (D). "P" and "T" on the Penrose drain coursing under the sciatic nerve refer to peroneal and tibial components of the sciatic nerve, respectively.

MRI, which showed a right sciatic mass near the sciatic notch that measured $4 \mathrm{~cm}$ in diameter (Fig. 3A). On physical examination, she had full motor strength, normal sensation, normal reflexes, and a negative straight leg raise test.

Preoperatively, ultrasound-guided needle electrical stimulation was used to localize her mass in the right posterior buttock region (Fig. 3B). After this localization, MB was administered as described above (Fig. 3C). A $15-\mathrm{cm}$ incision was centered over the mark from the ultrasound injection (Fig. 3C) and the MB tract was followed to the mass. A 4-cm mass was noted adjacent to the right sciatic nerve, and this was subsequently resected (Fig. 3D). The final pathology was a benign spindle cell neoplasm, compatible with a diagnosis of myxoma (Fig. 3E). Her right buttock pain and tenderness resolved following the surgery.

\section{Discussion}

The first study to use MB as an operative tool for peripheral nerve surgery, administered using ultrasound guidance, was by Gofeld et al. in 2013. ${ }^{6}$ Although that 

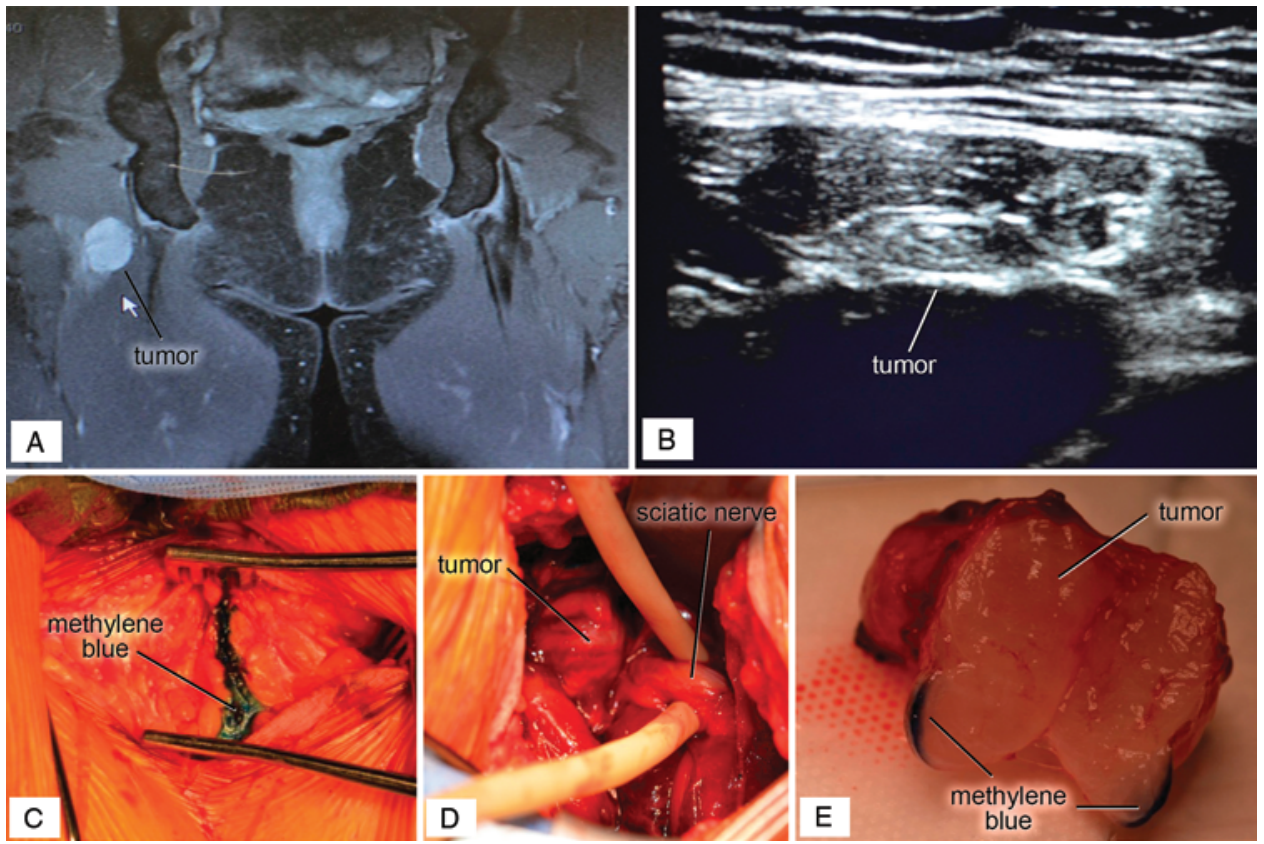

FIG. 3. Case 7. A patient with a sciatic myxoma. A: The MR neurogram is shown; the arrow indicates the tumor. B: The ultrasound image that was used for administration of $M B$ is shown with the tumor in view. $C$ and $D$ : Intraoperative photographs of the sciatic myxoma. Shown in $\mathrm{C}$ is the initial subcutaneous dissection demonstrating the MB tract. Immediately prior to removal of the tumor a photograph was taken to show the tumor and normal sciatic nerve (D). E: After the tumor was removed, the borders of the tumor are shown with peripheral staining of MB.

study did show the benefit of using ultrasonography and MB for localizing peripheral nerves, specifically for operations, it did not include a confirmatory test that could be performed prior to administration of MB to validate the intended nerve that was being localized. This study has this added dimension and security for localizing nerves, and that is the use of the ultrasound-guided cannulated needle, which provided nerve stimulation for further confirmation of the intended nerve target. Previously, Thomassen et al. in 2013 described the use of ultrasound guidance and nerve-stimulator guided blocks for neurectomy. ${ }^{21}$ In this paper we present a novel technique that provides the peripheral nerve surgeon a helpful tool to plan and execute the surgery. It is a technique that uses readily available, portable, and cost-effective resources that could be easily implemented to the benefit of many patients with a wide range of peripheral nerve problems.

The added benefit of nerve stimulation is unique to this study. It provides a confirmatory motor or sensory response that can be appreciated prior to the administration of MB. This confirmatory variable could potentially prevent incorrectly identifying a target nerve on preoperative ultrasonography. Also, as shown in Fig. 1D and E, the nerve stimulation response that is being monitored by the neurophysiologist provides direct feedback about the distance between the needle and the target nerve.

Furthermore, ultrasonography may have additional roles in the perioperative and intraoperative management of peripheral nerve disease. Ultrasound mapping of nerve pathology may enable delineation of normal structures in relation to nerve pathology, which may assist in planning the optimal surgical approach. As an example, ultrasound may be used to identify the location of normal nerve fascicles in relation to peripheral nerve sheath tumors, thus minimizing the chance of iatrogenic nerve injury. ${ }^{20}$ The addition of MB staining of the chosen surgical path may further improve the safety and efficiency of the surgical procedure. Although there have been few publications stating concern for the safety of MB use in patients, ${ }^{8}$ this has been easily addressed in this technique by using a dilute concentration of MB at low volumes. Similarly, ultrasound mapping and guidance may enable safe and accurate percutaneous needle biopsies of suspected peripheral nerve sheath tumors. ${ }^{2}$

Intraoperative ultrasonography applied to surgically exposed nerve lesions may provide the surgeon with another tool to evaluate nerve pathology, such as the extent of peripheral nerve injury following trauma. ${ }^{14}$ This could be further applied to peripheral nerve sheath tumor resection as a complement to the surgical microscope and intraoperative electrophysiology apparatus, to provide information regarding the internal characteristics of the tumor and its relationship to nerve structures, thereby possibly increasing the potential for normal nerve salvage.

Finally, ultrasound-guided near-nerve neurography may also be useful in the diagnostic workup of peripheral nerve lesions, particularly when evaluating early regeneration after peripheral nerve trauma. ${ }^{3}$ Presently, intraoperative compound nerve action potentials are recorded by exposing the nerve and applying electrodes directly to the nerve. Introducing electrodes next to the nerve using ultrasoundguidance techniques is a noninvasive option that may obviate the need for surgical exploration. 


\section{Conclusions}

Preoperative ultrasound/stimulation-guided MB administration is a promising technique that peripheral nerve surgeons could use to plan and execute surgery.

\section{References}

1. Bodner G, Bernathova M, Galiano K, Putz D, Martinoli C, Felfernig M: Ultrasound of the lateral femoral cutaneous nerve: normal findings in a cadaver and in volunteers. Reg Anesth Pain Med 34:265-268, 2009

2. da Costa RC, Parent JM, Dobson H, Ruotsalo K, Holmberg $\mathrm{D}$, Duque MC, et al: Ultrasound-guided fine needle aspiration in the diagnosis of peripheral nerve sheath tumors in 4 dogs. Can Vet J 49:77-81, 2008

3. de Kool BS, Blok JH, Walbeehm ET, van Neck JW, Hovius SE, Visser GH: Ultrasound-guided near-nerve neurography for early evaluation of nerve regeneration. J Neurosci Methods 174:265-271, 2008

4. Ernberg LA, Adler RS, Lane J: Ultrasound in the detection and treatment of a painful stump neuroma. Skeletal Radiol 32:306-309, 2003

5. Fischler AH, Gross JB: Ultrasound-guided sciatic neuroma block for treatment of intractable stump pain. J Clin Anesth 19:626-628, 2007

6. Gofeld M, Bristow SJ, Chiu S, Kliot M: Preoperative ultrasound-guided mapping of peripheral nerves. J Neurosurg 119:709-713, 2013

7. Gofeld M, Christakis M: Sonographically guided ilioinguinal nerve block. J Ultrasound Med 25:1571-1575, 2006

8. Hackett ER, Kline DG: Methylene blue. Surg Neurol 50:608-609, 1998

9. Harari A, Sippel RS, Goldstein R, Aziz S, Shen W, Gosnell $\mathrm{J}$, et al: Successful localization of recurrent thyroid cancer in reoperative neck surgery using ultrasound-guided methylene blue dye injection. J Am Coll Surg 215:555-561, 2012

10. Horn JL, Pitsch T, Salinas F, Benninger B: Anatomic basis to the ultrasound-guided approach for saphenous nerve blockade. Reg Anesth Pain Med 34:486-489, 2009

11. Huntoon MA, Burgher AH: Ultrasound-guided permanent implantation of peripheral nerve stimulation (PNS) system for neuropathic pain of the extremities: original cases and outcomes. Pain Med 10:1369-1377, 2009

12. Huntoon MA, Hoelzer BC, Burgher AH, Hurdle MF, Huntoon EA: Feasibility of ultrasound-guided percutaneous placement of peripheral nerve stimulation electrodes and anchoring during simulated movement: part two, upper extremity. Reg Anesth Pain Med 33:558-565, 2008

13. Jose J, Smith MK, Kaplan LD, Lesniak BP, Levi AD: Ultrasound-guided needle localization of the saphenous nerve for removal of neuroma in the infrapatellar branches: technical report. Neurosurgery 75:717-722, 2014

14. Koenig RW, Schmidt TE, Heinen CP, Wirtz CR, Kretschmer $\mathrm{T}$, Antoniadis $\mathrm{G}$, et al: Intraoperative high-resolution ultra- sound: a new technique in the management of peripheral nerve disorders. J Neurosurg 114:514-521, 2011

15. Lam NC, Petersen TR, Gerstein NS, Yen T, Starr B, Mariano ER: A randomized clinical trial comparing the effectiveness of ultrasound guidance versus nerve stimulation for lateral popliteal-sciatic nerve blocks in obese patients. J Ultrasound Med 33:1057-1063, 2014

16. Lee FC, Singh H, Nazarian LN, Ratliff JK: High-resolution ultrasonography in the diagnosis and intraoperative management of peripheral nerve lesions. J Neurosurg 114:206-211, 2011

17. Nahlieli O, Levy Y: Intravital staining with methylene blue as an aid to facial nerve identification in parotid gland surgery. J Oral Maxillofac Surg 59:355-356, 2001

18. Ryan WR, Orloff LA: Intraoperative tumor localization with surgeon-performed ultrasound-guided needle dye injection. Laryngoscope 121:1651-1655, 2011

19. Seif C, Martínez Portillo FJ, Osmonov DK, Böhler G, van der Horst C, Leissner J, et al: Methylene blue staining for nerve-sparing operative procedures: an animal model. Urology 63:1205-1208, 2004

20. Simon NG, Cage T, Narvid J, Noss R, Chin C, Kliot M: High-resolution ultrasonography and diffusion tensor tractography map normal nerve fascicles in relation to schwannoma tissue prior to resection. J Neurosurg 120:1113-1117, 2014

21. Thomassen I, van Suijlekom JA, van de Gaag A, Ponten JE, Nienhuijs SW: Ultrasound-guided ilioinguinal/iliohypogastric nerve blocks for chronic pain after inguinal hernia repair. Hernia 17:329-332, 2013

\section{Disclosure}

The authors report no conflict of interest concerning the materials or methods used in this study or the findings specified in this paper.

\section{Author Contributions}

Conception and design: Kliot, Osorio, Breshears, Aleshi. Acquisition of data: Kliot, Osorio, Breshears, Simon, HastingsRobinson, Aleshi. Analysis and interpretation of data: Kliot, Osorio. Drafting the article: Kliot, Osorio, Breshears, Arnaout, Hastings-Robinson. Critically revising the article: Kliot, Osorio, Breshears, Arnaout, Simon, Hastings-Robinson. Reviewed submitted version of manuscript: Kliot, Osorio, Arnaout, Simon, Aleshi. Approved the final version of the manuscript on behalf of all authors: Kliot. Statistical analysis: Osorio. Administrative/ technical/material support: Osorio. Study supervision: Kliot, Osorio.

\section{Correspondence}

Michel Kliot, Department of Neurological Surgery, Northwestern University Feinberg School of Medicine, 676 N. St. Clair St., Ste. 2210, Chicago, IL 60611. email: michelkliot@gmail.com. 